\title{
Current Status of Endovascular Repair of Aortic Dissections
}

\section{S Sharma}

\section{Department of Cardiac Radiology, All India Institute of Medical Sciences, New Delhi}

\begin{abstract}
Aortic dissection is a medical emergency and can quickly lead to death, even with optimal treatment. The Stanford classification is widely used and is in close relationship to clinical practice, as type A dissections require primary surgical repair whereas type B dissections are treated medically as initial treatment with surgery or endovascular repair reserved for any complications. Multi-slice CT is fast emerging as the investigation of choice to establish the diagnosis and plan treatment strategies in aortic dissection. The therapeutic strategies differ for treatment of an acute dissection compared to a chronic dissection. Most institutions favor a 'complication specific' approach for type B dissections with medical anti-hypertensive treatment and the use of beta-blockers as the primary therapy. Surgery or endovascular repair is reserved for patients with recurrent pain, life-threatening complications or rapid aortic expansion. Procedural success during endovascular repair for type B aortic dissection is reported in $99.2 \pm 0.1 \%$ of patients. Overall survival rates of $96.9 \%$ at 30 days, $96.7 \%$ at 6 months, $96.4 \%$ at 1 year, $95.6 \%$ at 2 years, and $95.2 \%$ at 5 years are reported after endovascular repair in Type B aortic dissections. There is evidence that endovascular repair may be non-inferior to surgery in this group of patients. Device designs and management algorithms are still evolving. More validated clinical data is necessary to define the role of endovascular repair in the management of type B aortic dissections.
\end{abstract}

Aortic dissection occurs when a tear in the inner wall of the aorta causes blood to flow between the layers of the wall of the aorta and force the layers apart. The dissection typically extends in an ante grade fashion, but can also extend in a retrograde manner from the site of the intimal tear. Aortic dissection is a medical emergency and can quickly lead to death, even with optimal treatment. It is one of the most catastrophic events that can affect the aorta and is a one of the two conditions in which the aorta grows very rapidly over a short time. The mortality rate in patients with untreated acute aortic dissection increases by $1 \%$ per hour, with $13 \%$ of patients dying by 12 hours, $21 \%$

Correspondence to: Dr. Sanjiv Sharma

Department of Cardiac Radiology, All India

Institute of Medical Sciences, New Delhi

E-mail: meetisv@yahoo.com by 24 hours and approximately $80 \%$ are dead within 2 weeks ${ }^{1-4}$. It is about 2-3 times more common than a ruptured abdominal aortic aneurysm.

\section{Classification systems}

Various methods have been used to describe aortic dissections. The systems commonly in use are either based on the anatomy of the dissection or the duration of onset of symptoms prior to presentation 5 . The Stanford classification divides dissections into two types depending on whether the ascending aorta is involved. Type A involves the ascending aorta whereas type B involves the descending aorta distal to the left subclavian artery. The DeBakey classification divides dissections into three types based on where the original intimal 
tear is located and the extent of the dissection. These include:

- Type I involves the ascending aorta, aortic arch, and descending aorta

- Type II is confined to the ascending aorta

- Type III is confined to the descending aorta distal to the left subclavian artery

Type III dissections are further divided into IIIa and IIIb. Type IIIa refers to dissections that originate distal to the left subclavian artery but extend proximally and/or distally, mostly above the diaphragm. Type IIIb refers to dissections that originate distal to the left subclavian artery, extend only distally and may extend below the diaphragm. Svensson classification is more etiology and image morphology based, and divides this group of abnormalities into five classes:

Class 1: Classic dissection with true and false lumen

Class 2: Intramural hematoma or hemorrhage Class 3: Subtle dissection without hematoma Class 4: Atherosclerotic penetrating ulcer Class 5: Iatrogenic or traumatic dissection

The Stanford classification is in close relationship to clinical practice, as type A dissections require primary surgical repair whereas type B dissections are treated medically as initial treatment with surgery or endovascular repair reserved for any complications.

The causes of aortic dissection include, among others ${ }^{1-5}$ :

1. Hypertension- $72-80 \%$ patients have associated hypertension

2. Connective tissue disorders

3. Marfan's syndrome; Turner's syndrome; seudoxanthoma elasticum

4. Vasculitis

5. Trauma- Accidental or iatrogenic, such as after catheterization
6. Late sequale of cardiac surgery, especially after aortic valve replacement for aortic regurgitation

7. Bicuspid aortic valve

8. Tertiary syphilis

Typically, aortic dissection is seen in the 5070 year age group and is twice as common in men. In young women, over half the cases are seen during pregnancy, typically in the third trimester or in the post-partum period. Type B dissection accounts for approximately $40 \%$ of all dissections and typically occurs in the morning period, more commonly in winters. The clinical diagnosis is often difficult to establish as the symptoms can mimic a variety of diseases, including myocardial infarction and pericarditis, among others.

Imaging: Some form of imaging is usually necessary to establish the diagnosis, define the extent of dissection and involvement of branch vessels and identify the complications. The selection of the imaging technique is usually based on the pre-test likelihood of the diagnosis, availability of the testing modality, patient stability, and the sensitivity and specificity of the test. Multislice CT is fast emerging as the investigation of choice to establish the diagnosis and plan treatment strategies in aortic dissection ${ }^{7,8}$. It is preferred over other imaging techniques due to its speed, reproducibility, high spatial \& contrast resolution and its orthogonal potential (ability to provide the best anatomic plane for sizing of proximal and distal neck and landing zones). However, two issues, including the radiation dose and its potential for nephro-toxicity, should be kept in mind while using this technique as these patients will often need a catheter angiography and endovascular treatment in the immediate period and many imaging studies during follow up. Usually, a dualphase CTA is recommended in the preoperative evaluation. A typical protocol should include imaging before administration of contrast and imaging after 
contrast administration in the arterial phase. Imaging in post-contrast delayed phase is unnecessary in the pre-operative period but must always be done in the follow up after endovascular treatment to detect the endoleaks. MRI can also be used for establishing the diagnosis but is less reliable in terms of measurements for device sizing for endovascular repair. We use duplex ultrasound for the evaluation and measurement of the access vessels in the groin.

Some pertinent issues related to imaging interpretation, including the influence of inherent level of resolution of current imaging technologies on size assessment, the relevance of inclusion or exclusion of the aortic wall in the measurement of the device and sac size, the limitations of specific imaging modalities in terms of profiling the various anatomic segments of the aorta, the influence of geometric complexity of the aorta on the diagnosis and device sizing, the accuracy of comparison of serial images showing 1-2 $\mathrm{mm}$ change in size over two studies, changes in the shape of the aorta as a marker of disease, the detection of disease activity and its influence on the sac diameters, variations in the aortic diameter during systole and diastole and their impact on size measurements, and factoring in the body surface area while relating aortic size to the hinge points for management decision making, among others should be kept in mind while evaluating imaging studies for the diagnosis and management of aortic dissections ${ }^{5-8}$.

The CT images should be analyzed to identify the length of the residual aortic tissue above \& below the false channel aneurysm (at least 1.5-2 cm length of normal aorta devoid of any branches above and below the intended site of device delivery should be available for endovascular repair to succeed); the relation of the dissection and false channel aneurysm to the branch vessels, obstructions of the aortic branch vessels (fixed versus dynamic) and the amount of calcium \& thrombus in the diseased segment. At least one common iliac artery should be preferably free of dissection for use as the access vessel for successful endovascular repair. It is also important to ensure that adequate diameter vascular access (external iliac or common femoral artery of $>7.5 \mathrm{~mm}$ diameter) free of dissection, extreme tortuosity, extensive calcification and obstructive disease is available for the device delivery.

Choice of Treatment: In an acute dissection (within the first two weeks of the appearance of symptoms), the treatment choice depends on its location. For Stanford type A dissection, surgical management is preferred. For uncomplicated Stanford type B dissections, medical management is preferred in the initial period. The risk of death due to aortic dissection is highest in the first few hours after the dissection begins, and decreases afterwards. As a result, the therapeutic strategies differ for treatment of an acute dissection compared to a chronic dissection. If the patient survives this period, the prognosis is improved. Approximately, two-thirds of all dissections present in the acute phase. The patients who present two weeks after the onset of dissection are said to have chronic aortic dissections. These individuals have been self-selected as survivors of the acute episode, and can be treated with medical therapy as long as they are stable and free of complications. Complicated type $\mathrm{B}$ dissections can be treated by surgical or endovascular repair ${ }^{9-}$ 14

The indications for endovascular or surgical repair of Type B dissection include the following ${ }^{15-18}$ :

* Aortic \& false lumen diameters-

\# Total aortic diameter $>6 \mathrm{~cm}$ (there is emerging recent evidence of increased mortality in $>40 \mathrm{~mm}$ diameter)

\# Total diameter of $4.5 \mathrm{~cm}$ may be used for cut-off in asymptomatic patients with connective tissue disorders

\# Absolute false lumen diameter > $22 \mathrm{~mm}$ 
\# Cross- sectional area of false lumen divided by area of whole aorta- $\geq 0.7$

\# In symptomatic patients, the treatment should be offered irrespective of the aortic diameter * Patent primary entry site * Expanding false lumen * Recurrent chest or back pain * End-organ ischemia due to branch vessel involvement or claudication

* Refractory hypertension

\section{Case selection}

The anatomic criteria for suitability of endovascular repair include the presence of an optimal $(>1.5-2 \mathrm{~cm})$ proximal and distal landing zone away from any major branch, such as the left subclavian and the celiac artery; presence of at least one iliac artery free of dissection for access, relatively straight vascular access in the aorta and the pelvic arteries free of gross atheromatous disease, and an adequate access vessel diameter (>7-8 $\mathrm{mm}$ depending on the device size). Patients with evidence of infection, previous ilio-femoral stenting, contrast allergies, renal insufficiency, poor distal runoff vessels, extensive aortic calcification or circumferential thrombus in landing zones, extremely tortuous vascular anatomy or with significant obstructive disease in access route, among others are relative contraindications for this treatment.

\section{Device selection}

The optimal diameter of the stent-graft should be matched to the diameter of the normal aorta proximal to the dissection. Minimal over sizing of the graft is necessary. At most, there should be $<10 \%$ over sizing. This minimizes the chance for graft infolding and limits its ability to cause damage to the normal aorta from its radial force. The graft diameter should be sized to the proximal normal aorta and that diameter should be carried distally in the stent-graft despite the diameter of the distal true lumen. Retrograde filling of the false lumen from a distal fenestration in the abdominal aorta is acceptable and should not be considered a failure of the therapy. The purpose of the therapy is to seal all significant fenestrations in the thoracic aorta. Frequently, this is only the proximal entry tear. When there are other fenestrations, they should be covered. If the true lumen distal to the stent-graft is still significantly compressed, then an additional stent-graft component or bare stents can be added to the proximal stent-graft, to provide mechanical support to distend the collapsed true lumen.

\section{Management strategies}

At present, most institutions favor a 'complication specific' approach for type B dissections with medical anti-hypertensive treatment and the use of beta-blockers as the primary therapy. Surgery or endovascular repair is reserved for patients with recurrent pain, life-threatening complications or rapid aortic expansion. The concept of endovascular stent-graft placement was propelled by the desire to induce aortic remodeling by sealing the proximal entry tear, at the same time avoiding the risks associated with open surgery. This rationale was originally based on the clinical observation that patients with spontaneous thrombosis of the false lumen have a better long-term prognosis than without. Conversely, persistent perfusion of the false lumen has been identified as an independent predictor of progressive aortic enlargement and adverse long-term outcome. Nevertheless, spontaneous thrombosis of the false lumen is a rare observation $(<4 \%$ of patients) and most often requires interventions to exclude it from the true lumen. It is unclear whether thrombosis of the false lumen and stabilization of the aortic diameter translates into long-term prevention of aortic rupture. Mid-term results suggest that there is adequate prevention of aneurysm rupture ${ }^{19-23}$. 
The advent of endovascular repair has changed the management algorithms in recent years ${ }^{15-23}$. The basic purpose of using stent-grafts is to completely cover the primary entry tear and to eliminate most of the inflow to the false lumen, thus promoting thrombosis of the false lumen. The graft serves to exclude flow through the initial tear in the intima and redirect aortic blood flow exclusively into the true lumen reestablishing perfusion of aortic branches affected by the spreading dissection. This can also avoid a true lumen collapse.

Procedural success during endovascular repair for type B aortic dissection is reported in $99.2 \pm 0.1 \%$ of patients. In one large metaanalysis reported recently, major complications were reported in $3.4 \pm 0.1 \%$ patients, with the most severe neurologic complications in $0.6 \%$ patients. Periprocedural stroke was encountered more frequently than paraplegia $(0.2 \%$ vs $0 \%)$. The overall 30 -day mortality was $2.6 \pm 0.1 \%$. In addition, $1.5 \pm 0.1 \%$ of the patients died over a mean follow-up period of $27.1 \pm 17.5$ months. Life-table analysis yielded overall survival rates of $96.9 \%$ at 30 days, $96.7 \%$ at 6 months, $96.4 \%$ at 1 year, $95.6 \%$ at 2 years, and $95.2 \%$ at 5 years ${ }^{19-23}$.

Overall, there is reasonable consensus in the literature that acute type A dissections should be treated by surgery and that uncomplicated type B dissections should be treated with optimized medical therapy. Complicated acute type B dissections should be offered treatment by endovascular repair in suitable patients. Surgery may be reserved for those in whom endovascular repair is not feasible, fails or results in a complication. Strategies for managing chronic complicated type B dissections are less clear. There is some evidence that endovascular repair may be non-inferior to surgery in this group of patients. It is worth mentioning here that the current devices used for endovascular repair are not designed for the treatment of aortic dissection and have major limitations in terms of addressing the requirements for optimal treatment goals. Overall, device designs and management algorithms in these patients are still evolving. More validated clinical data is necessary to define the role of endovascular repair in the management of type B aortic dissections.

\section{References}

1. Elefteriades JA, Rizzo JA. Epidemiology, prevalence, incidence, trends. In: Elefteriades JA, editor. Acute Aortic Disease. New York, NY: Informa Healthcare, 2008:89-98.

2. Mehta RH, Bossone E, Evangelista A, et al; on behalf of the International Registry of Acute Aortic Dissection (IRAD) Investigators. Acute type B aortic dissection in elderly patients: clinical features, outcomes and simple risk stratification rule. Ann Thorac Surg 2004;77:1622-1629.

3. Hagan PG, Nienaber CA, Isselbacher EM, et al. The International Registry of Acute Aortic Dissection (IRAD): new insights into an old disease. JAMA 2000;283:897-903.

4. Erbel R, Alfonso F, Boileau C,et al. Diagnosis and management of aortic dissection. Eur Heart J 2001;22:16421681.

5. Elefteriades JA, Farkas EA: Thoracic Aortic Aneurysm- Clinically Pertinent Controversies and Uncertainties. J Am Coll Cardiol 2010;55:841-57.

6. Nienaber CA, Eagle KA. Aortic dissection: new frontiers in diagnosis and management: Part II: therapeutic management and follow-up. Circulation 2003;108:772-778.

7. Muhs BE, Vincken KL, van Prehn J, et al. Dynamic cine-CT angiography for the evaluation of the thoracic aorta; 
insight in dynamic changes with implications for thoracic endograft treatment. Eur J Vasc Endovasc Surg 2006;32:532- 6 .

8. Ueda T, Fleischman, Rubin GD, Dake MD, Sze DY: Imaging of the thoracic aorta before and after stent-graft repair of aneurysms and dissections. Semin Thorac Cardiovasc Surg 2008;20(4):348-357.

9. Onitsuka S, Akashi H, Tayama K, Okazaki T, Ishihara K, Hiromatsu S, Aoyagi S: Long-term outcome and prognostic predictors of medically treated acute type B aortic dissections. Ann Thorac Surg 2004; 78(4):1268-73.

10. Akin I, Kische S, Ince H, Nienaber CA: Indication, timing and results of endovascular treatment of type B dissection. Eur J Vasc Endovasc Surg 2009;37(3):289-96

11. Schneider AJ. Assessment of risk factors and surgical outcome. Surg Clin North Am 1983;63:1113-1126.

12. Feldman M, Shah M, Elefteriades JA. Medical management of acute type A aortic dissection. Ann Cardiovasc Thorac Surg 2009;15: 286-93.

13. Walsh SR, Tang TY, Sadat U, Naik J, Gaunt ME, Boyle JR, Hayes PD, Varty $\mathrm{K}$ : Endovascular stenting versus open surgery for thoracic aortic disease: systematic review and meta-analysis of perioperative results. J Vasc Surg. 2008;47(5):1094-1098.

14. Coady MA, Rizzo JA, Hammond GL, Kopf GS, Elefteriades JA. Surgical intervention criteria for thoracic aortic aneurysms: a study of growth rates and complications. Ann Thorac Surg 1999;67:1922-6.
15. Juvonen T, Ergin MA, Galla JD,et al. Risk factors for rupture of chronic type B dissections. J Thorac Cardiovasc Surg 1999;117: 776-786.

16. Davies RR, Goldstein LJ, Coady MA Tittle SL, Rizzo JA, Kopf GS, Elefteriades JA: Yearly rupture or dissection rates for thoracic aortic aneurysms: simple prediction based on size. Ann Thorac Surg 2002; 73(1):1727.

17. Dake MD, Kato N, Mitchell RS, et al. Endovascular stent-graft placement for the treatment of acute aortic dissection. N Engl J Med 1999;340:1546-1552.

18. Shimono T, Kato N, Yasuda F,et al. Transluminal stent-graft placements for the treatments of acute onset and chronic aortic dissections. Circulation 2002;106:I241-I247.

19. Jonker FH, Trimarchi S, Verhagen HJ, Moll FL, Sumpio BE, Muhs BE: Metaanalysis of open versus endovascular repair for ruptured descending thoracic aortic aneurysm. J Vasc Surg 2010; 51(4):1026-32.

20. Nienaber CA, Rousseau H, Eggebrecht $\mathrm{H}$, et al: Randomized comparison of strategies for type B aortic dissection: the INvestigation of STEnt Grafts in Aortic Dissection (INSTEAD) trial. Circulation 2009;120(25):2519-28.

21. Parsa CJ, Schroder JN, Daneshmand MA, McCann RL, Hughes GC: Midterm results for endovascular repair of complicated acute and chronic type B aortic dissection. Ann Thorac Surg 2010;89(1):97-102.

22. Xiong J, Jiang B, Guo W, Wang SM, Tong XY: Endovascular stent graft placement in patients with type $\mathrm{B}$ aortic 
dissection: a meta-analysis in China. J Thorac Cardiovasc 2009;138(4):865-72.
23. Eggebrecht H, Nienaber CA, Neuhäuser $M$, et al: Endovascular stent-graft placement in aortic dissection: a metaanalysis. Eur Heart J 2006; 27(4):49-98. 